\title{
The effect of uraemia on the duration of arrhythmias in the context of cardioprotective ischaemic conditioning strategies
}

\author{
Kieran McCafferty, Conor J Byrne, Julius Kieswich, Martin Raftery, \\ Christoph Thiemermann, Muhammad M Yaqoob
}

\begin{abstract}
- Additional material is available. To view please visit the journal online (http://dx. doi.org/10.1136/heartasia2013-010432)
\end{abstract}

Department of Translational Medicine and Therapeutics, William Harvey Research Institute, Queen Mary University London, London, UK

\section{Correspondence to} Dr Kieran McCafferty, Department of Translational Medicine and Therapeutics, William Harvey Research Institute, John Vane Science Centre, Charterhouse Square, London EC1M 6BQ, UK: kieranmccafferty@gmail.com

$\mathrm{KM}$ and $\mathrm{CJB}$ contributed equally to the body of work presented in this manuscript and are joint first authors.

Received 4 September 2013 Revised 15 April 2014 Accepted 2 May 2014
CrossMark

\footnotetext{
To cite: McCafferty K, Byrne $C J$, Kieswich J, et al. Heart Asia 2014;6:76-82. doi:10.1136/heartasia-2013010432
}

\begin{abstract}
Background Sudden cardiac death is a leading cause of death in patients with chronic kidney disease and end stage renal disease. Ischaemic conditioning strategies confer profound myocardial protection and, in the absence of uraemia, have been reported to reduce myocardial dysrhythmias. Recent data confirms that ischaemic conditioning can protect the uraemic heart. However, the effect of uraemia on myocardial arrhythmogenesis in the context of ischaemia-reperfusion injury and whether ischaemic conditioning can modulate this is unknown.

Objective We investigated the effect of underling chronic uraemia on the duration of arrhythmias in the context of cardioprotective ischaemic conditioning strategies.
\end{abstract}

Methods We examined the effect of chronic uraemia on arrhythmias occurring in the context of myocardial ischaemia-reperfusion injury and the ability of ischaemic preconditioning (IPC), remote ischaemic preconditioning (RIPC) and ischaemic postconditioning (iPOST) to suppress arrhythmogenesis in uraemic and non-uraemic animals.

Results IPC led to a reduction in the frequency and duration of arrhythmias occurring during ischaemia and reperfusion. Neither RIPC nor iPOST affected the duration or frequency of ischaemic or reperfusion arrhythmias. Underlying uraemia did not alter the frequency or duration of ischaemic arrhythmias in any of the experiments however it was associated with a reduction in reperfusion arrhythmia duration in the IPC and IPOST experiments.

Conclusions These studies demonstrate that underlying chronic uraemia does not reduce the threshold for arrhythmia timing or duration resulting from myocardial ischaemia-reperfusion and underlying uraemia did not alter the effects of these cardioprotective ischaemic conditioning strategies in the context of arrhythmia duration.

Summary This novel work in a rodent model of chronic uraemia establishes that underlying uraemia does not increase the susceptibility to myocardial ischaemiareperfusion induced arrhythmias. When compared with the non-uraemic heart, the uraemic heart has a similar response to the effects of ischaemic conditioning strategies in terms of their effect on arrhythmia timing and duration.

\section{INTRODUCTION}

Cardiovascular disease is the leading cause of death for patients with moderate to severe chronic kidney disease. ${ }^{1}$ Sudden cardiac death/arrhythmia represents the most common cause of cardiovascular mortality in end stage renal disease, with $26 \%$ of deaths attributable to cardiac arrhythmias. ${ }^{1}$

Structural and functional abnormalities of the cardiovascular system such as left ventricular hypertrophy (LVH), myocardial fibrosis, inflammation, sympathetic overactivity and electrolyte disturbances, along with a clustering of classical risk factors for arrhythmias such as old age, ischaemic heart disease and diabetes affect individuals with chronic uraemia to create a 'perfect storm' for cardiac dysrhythmias. ${ }^{1-5}$

Ischaemic conditioning strategies encompass a number of tissue protective techniques, described over the last quarter of a century. Ischaemic preconditioning (IPC) was described by Murry et $a l^{6}$ who reported that brief episodes of 'sublethal' ischaemia followed by reperfusion, could confer resistance to a subsequent lethal episode of ischaemia-reperfusion injury (IRI). Subsequently Przyklenk et $a l^{7}$ reported that vascular beds adjacent to the preconditioned territory were also rendered resistant to IRI and the term remote ischaemic preconditioning (RIPC) was coined to describe this phenomenon. Lastly Zhao et $a l^{8}$ reported the phenomenon of ischaemic postconditioning (iPOST) whereby interruption of reperfusion by additional episodes of brief IRI led to a reduction in infarct size. In addition to their cytoprotective effect, the antiarrhythmic effect of IPC, RIPC and iPOST have also been investigated. IPC has been widely reported to reduce ischaemic ${ }^{9-11}$ and reperfusion $^{9} 12$ arrhythmias in animals. The antiarrhythmic effect of IPC has also been replicated in humans. ${ }^{13-15}$ However not all groups have demonstrated a beneficial effect of IPC on arrhythmogenesis. Grund et al ${ }^{16}$ reported that IPC increased arrhythmias in pigs and Jebeli et $a l^{17}$ found that IPC did not suppress arrhythmias in a cohort of patients undergoing elective coronary artery bypass (CABG).

RIPC also appears to suppress arrhythmias, however it is unclear whether RIPC suppresses only ischaemic arrhythmias, ${ }^{18}$ only reperfusion arrhythmias $^{19}$ or both. $^{20} 21$ iPOST has also been shown to reduce reperfusion arrhythmias in animals $^{22} 23$ and humans. ${ }^{24}$

Patients with chronic kidney disease (CKD) have been routinely excluded from clinical trials examining ischaemic conditioning induced cardioprotection. The impact of chronic uraemia on myocardial arrhythmias associated with IRI and the effect of ischaemic conditioning strategies on ischaemic and 
reperfusion arrhythmias in the uraemic heart are unknown. We analysed data on arrhythmia frequency and duration recorded during our previously published work on ischaemic conditioning in uraemic rats. ${ }^{25}$

\section{METHODS}

All experiments were approved by our institutional ethics committee and performed under license granted by The Home Office (UK) in accordance with the Animals (Scientific Procedures) Act 1986. Male Wistar rats were used for all experiments (Charles River Laboratories UK, Margate, UK).

\section{SUBTOTAL NEPHRECTOMY}

Animals underwent a two-stage subtotal nephrectomy (SNx) or a sham procedure as previously described. ${ }^{25}$ The animals were allowed 4 weeks following the second stage (right total nephrectomy) to recover and develop the uraemic phenotype before undergoing myocardial ischaemia-reperfusion.

\section{MYOCARDIAL ISCHAEMIA-REPERFUSION}

Myocardial ischaemia-reperfusion was carried out as previously described. ${ }^{25}$ Briefly, animals were anaesthetised, a tracheostomy was performed and the animal ventilated using small animal ventilator. A venous and an arterial line were inserted, to monitor pulse and blood pressure.

Following a left parasternal incision a $6 / 0$ silk suture was placed through the myocardium at the approximate level of the left anterior descending artery. A piece of polythene tubing was placed over the free ends of the suture to form a snare for reversible arterial occlusion.

\section{CONDITIONING PROTOCOLS}

\section{Ischaemic preconditioning}

One or three cycles of $5 \mathrm{~min}$ left anterior descending artery (LAD) occlusion (ischaemia) was followed by $5 \mathrm{~min}$ reperfusion before either $25 \mathrm{~min}$ or $35 \mathrm{~min}$ of sustained ischaemia (figure 1).
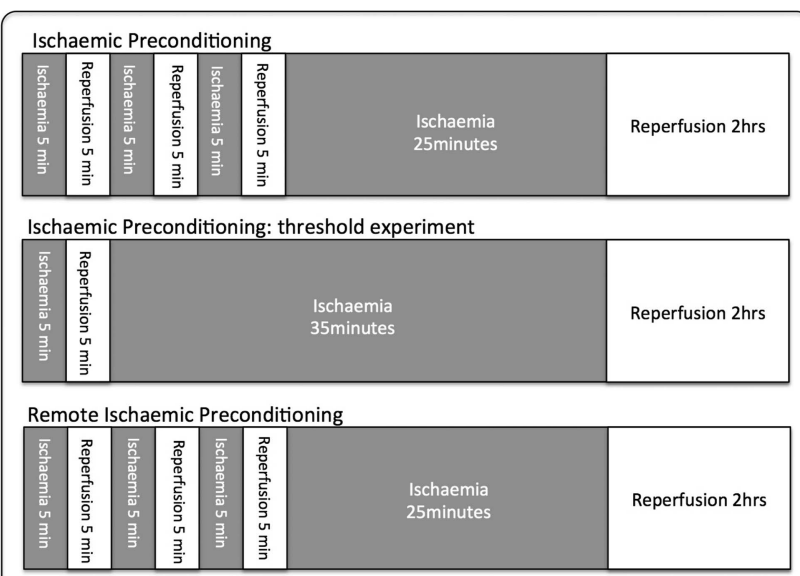

Ischaemic Postconditioning

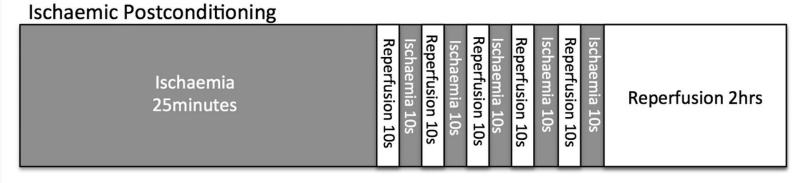

Figure 1 Diagrammatic representation of the conditioning protocols.

\section{Remote preconditioning}

The left femoral artery was dissected out from the femoral vein and nerve. A microvessel clip was used to occlude the artery. Pallor and a reduction in the temperature of the paw confirmed occlusion. Reperfusion was confirmed by hyperaemia followed by restoration of normal colour and temperature. Three cycles of $5 \mathrm{~min}$ ischaemia followed by $5 \mathrm{~min}$ reperfusion were employed.

\section{Postconditioning}

LAD occlusion (ischaemia) for $25 \mathrm{~min}$ with five cycles of $10 \mathrm{~s}$ reperfusion/10 s ischaemia upon reperfusion was followed by a further $1 \mathrm{~h} 58 \mathrm{~min}$ and $20 \mathrm{~s}$ reperfusion (ie, $2 \mathrm{~h}$ reperfusion in total).

At the end of the final reperfusion period the animals were sacrificed and the heart harvested for determination of area at risk and infarct size (see our previous work ${ }^{25}$ for results of the tissue protective effects of myocardial conditioning strategies in the context of chronic uraemia).

\section{Measurement of arrhythmias}

During the experiments all animals had continuous monitoring of heart rate and mean arterial pressure (MAP) using the Powerlab/85p system (ADInstruments).

Because the Powerlab/85p system displayed pressure waveforms for each heart beat, alterations in the MAP was used as a marker for the presence of arrhythmias. To confirm that alterations in the recorded MAP corresponded to arrhythmias, we performed contemporaneous ECG recordings (figure 2) on a subset of the animals $(n=12)$. The 3-lead ECG recording was captured by attaching electrodes to the left and right sides of the chest wall along with the left footpad. These tracings were displayed in real time along with the blood pressure waveforms and heart rate through LabChart software. This confirmed that myocardial arrhythmias led to alterations in cardiac output, which was visible through beat-to-beat MAP waveforms.

For the purposes of analysis, an arrhythmia was defined as a haemodynamic disturbance in the MAP trace waveform lasting longer than $2 \mathrm{~s}$. This time period was chosen so as to exclude occasional ectopic beats, which were unlikely to alter tissue perfusion.

Arrhythmias occurring during the first $30 \mathrm{~s}$ following LAD occlusion were excluded from analysis, as brief arrhythmias were not uncommon immediately after occlusion. These were often due to repositioning of the heart in the thoracic cavity using forceps to get good visualisation of the area at risk, rather than due to ischaemic arrhythmias.

The number and duration of arrhythmias (including cardiac arrest) occurring during the conditioning procedures, reperfusion or during the index ischaemia were recorded (figure 3 ). During the study, in response to a myocardial arrhythmia, no antiarrhythmic drugs were administered nor mechanical defibrillation manoeuvres performed.

\section{Statistical analysis}

Data was analysed with GraphPad Prism software (San Diego, California, USA). Given the non-normal distribution of the data set, non-parametric statistical analysis was used and the data are presented as median with IQR. A two-tailed Mann-Whitney U test with Dunn's postcomparison test were used to test for significance for all other experiments. In the RIPC and iPOST experiments, in addition to the Mann-Whitney $U$ test, a two-way analysis of variance (ANOVA) with Bonferroni post-test 


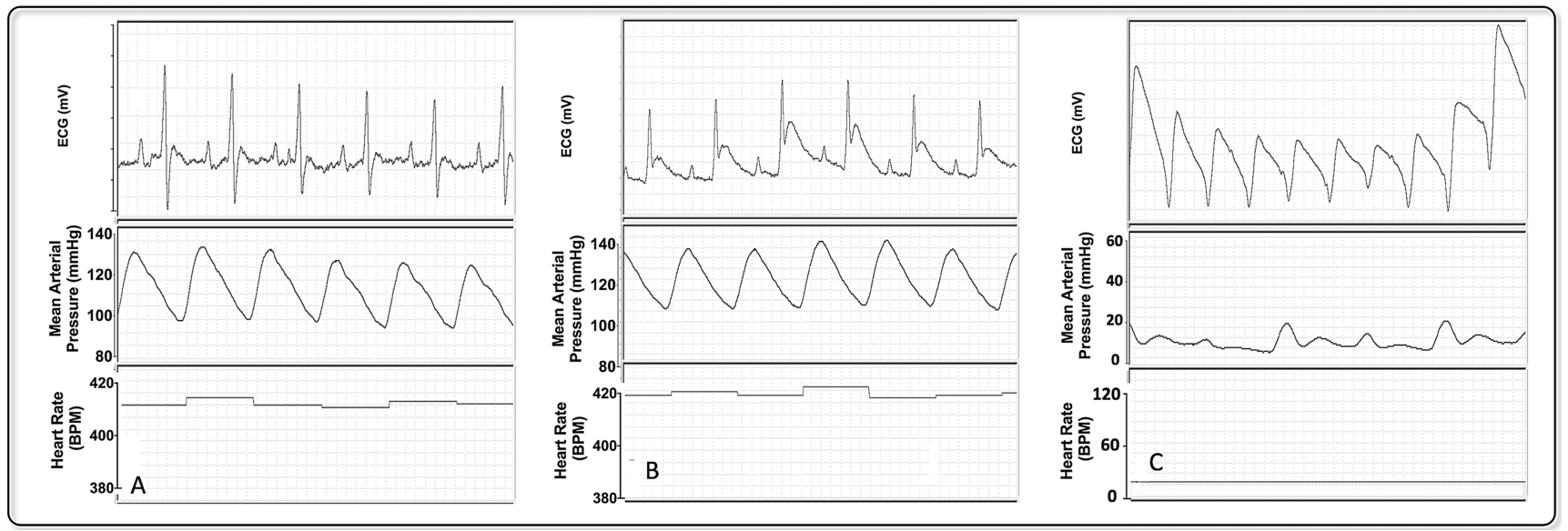

Figure 2 ECG tracing (top), mean arterial pressure (MAP) trace (middle) and heart rate trace (bottom) recordings during experiments. (A) Preocclusion. ECG demonstrates sinus rhythm with normal ST segments. (B) 3 min into ischaemia. ECG demonstrates ST segment elevation; the MAP trace is preserved with a regular heart rate. (C) 5 min into ischaemia ventricular tachycardia develops with loss of cardiac output manifested by loss in MAP trace and heart rate.

comparison were employed to analyse the interaction of uraemia with the conditioning protocol.

\section{RESULTS}

\section{Characterisation of the uraemic phenotype}

Four weeks following the second stage procedure, compared with sham operated animals, the uraemic animals were significantly growth restricted, hypertensive, anaemic and had an increased heart weight index (heart weight expressed as proportion of body weight); a surrogate for LVH (table 1). In addition the uraemic animals had over twice the serum creatinine and urea concentrations compared with sham operated animals (see online supplementary table for additional details on the characterisation of the model).

\section{Effect of three cycles of IPC on arrhythmia suppression in a model of chronic uraemia}

Three cycles of IPC led to a subsequent reduction in the frequency and duration of ischaemic arrhythmias: the median duration in the non-preconditioned and preconditioned groups was $25 \mathrm{~s}$ and $2 \mathrm{~s}$, respectively $(\mathrm{p}=0.003)$, with significantly fewer arrhythmias occurring in the preconditioned group $(\mathrm{p}<0.0001)$ (table 2). Three cycles of IPC also led to a reduction in the total duration and frequency of arrhythmias occurring after the 25 -min ischaemia (reperfusion arrhythmias): median duration in the non-preconditioned and preconditioned groups was $9 \mathrm{~s}$ and
$3 \mathrm{~s}$, respectively $(\mathrm{p}=0.001)$, with significantly fewer arrhythmias occurring in the preconditioned group $(\mathrm{p}=0.01)$. The total duration of all ischaemic arrhythmias (calculated as the sum of the duration of arrhythmias occurring during the ischaemic phase of preconditioning and arrhythmias occurring during the $25 \mathrm{~min}$ ischaemia) was reduced by the IPC protocol $(\mathrm{p}=0.03)$. The total duration of reperfusion arrhythmias (calculated as the sum of the arrhythmias occurring during the reperfusion phases of IPC and those occurring after the $25 \mathrm{~min}$ ischaemia) was increased by IPC protocol $(p=0.002)$, the increased reperfusion arrhythmias occurring during the IPC protocol meant that when all arrhythmias were considered together, the antiarrhythmic effect of IPC was lost $(p=0.87)$. In a similar way, when the total number of arrhythmias (including those occurring during IPC) were considered, the overall apparent reduction in the frequency of arrhythmias in the IPC group was lost $(p=0.09)$.

\section{Effect of underlying uraemia on arrhythmia suppression following a single IPC cycle}

Underlying uraemia did not alter the effect of IPC on the duration of arrhythmias occurring during the ischaemic phase of preconditioning $(p=0.23)$, or during $(p=0.74)$, or after $(p=0.64)$ the 35-min ischaemia period (table 3). However underlying uraemia was associated with a reduction in the duration of arrhythmias occurring during the reperfusion phase of preconditioning $(\mathrm{p}=0.02)$. This effect remained significant

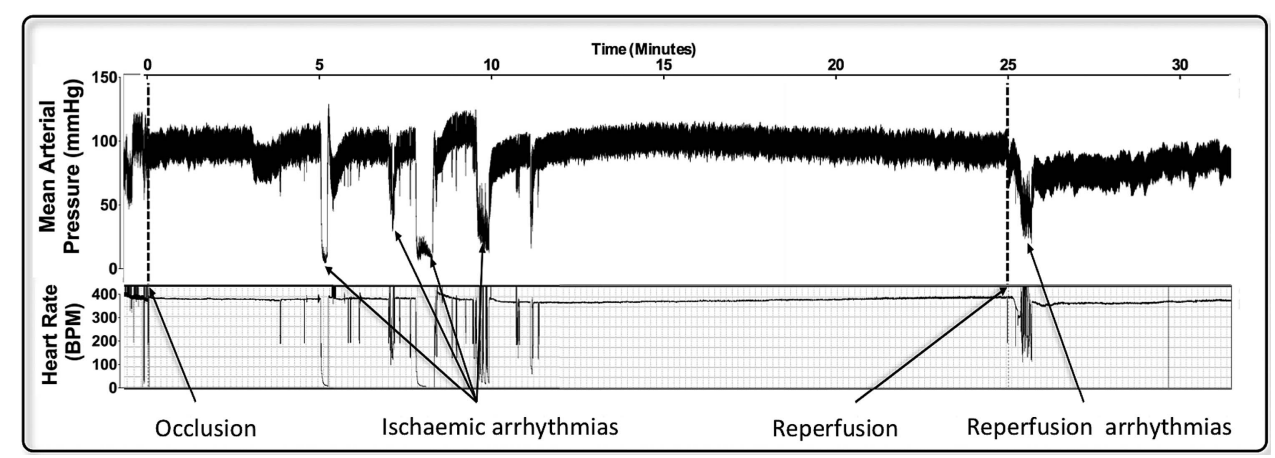

Figure 3 Mean arterial pressure (MAP) trace (top) and heart rate (bottom) during $25 \mathrm{~min}$ of ischaemia and early reperfusion. Arrhythmias are noted by a transient drop in the MAP and alteration in heart rate occurring 5-8 min post occlusion, and again shortly after reperfusion. 
Table 1 Characterisation of the uraemic phenotype

\begin{tabular}{lclc}
\hline & Sham SNx & SNx & p Value \\
\hline Weight (g) & $408(29.5)$ & $378(33.4)$ & 0.05 \\
Blood pressure (mm Hg) & $137(26)$ & $157(19.6)$ & 0.01 \\
Pulse rate (BPM) & $392(51.6)$ & $387(44.7)$ & 0.75 \\
Heart weight index* & $2.85(0.25)$ & $3.44(0.46)$ & 0.003 \\
Haematocrit (\%) & $37.8(4.51)$ & $27.1(5.06)$ & $<0.0001$ \\
Plasma bicarbonate (mmol/l) & $24.8(4.1)$ & $26.2(3.4)$ & 0.2 \\
Serum albumin (g/l) & $27.6(2.24)$ & $27.5(1.90)$ & 0.94 \\
Serum urea (mmol/l) & $6.2(1.34)$ & $17.4(4.61)$ & $<0.0001$ \\
Serum creatinine ( $\mu \mathrm{mol} / \mathrm{L})$ & $42.1(5.41)$ & $99.9(30.1)$ & $<0.0001$ \\
\hline
\end{tabular}

*The heart weight index was calculated by dividing the weight of the heart in grams by the weight of the animal in kilograms. Data displayed as mean (SD), with $p$ value calculated using an unpaired $t$ test.

when the duration of all reperfusion arrhythmias $(p=0.01)$ and all arrhythmias (ischaemic and reperfusion) were considered $(p=0.02)$. When the frequency of arrhythmias was considered, underlying uraemia did not appear to alter the number of arrhythmias occurring during the preconditioning phase $(\mathrm{p}=0.37)$, the 25 -min ischaemic phase $(\mathrm{p}=0.32)$ or the reperfusion phase $(\mathrm{p}=0.18)$.

\section{Effect of RIPC on arrhythmias in uraemic and non-uraemic animals}

RIPC had no significant effect on arrhythmia suppression in either uraemic or non-uraemic animals. There was no difference in the duration of ischaemic arrhythmias $(p=0.74)$, reperfusion arrhythmias $(\mathrm{p}=0.5)$ or the total duration of all arrhythmias $(p=0.54)$, nor was there any difference in the frequency of arrhythmias occurring during ischaemia $(\mathrm{p}=0.89)$ or reperfusion $(\mathrm{p}=0.64)$ (table 4). In addition no interaction was seen between underlying uraemia and RIPC in any of the above measurements using a two-way ANOVA.

\section{Effect of iPOST on arrhythmias in uraemic and non-uraemic animals}

Unsurprisingly an iPOST protocol had no effect on the duration $(p=0.36)$ or frequency $(p=0.27)$ of ischaemic arrhythmias as the procedure was only commenced at the point of reperfusion (table 5). iPOST appeared to lead to an increase in the duration of reperfusion arrhythmias $(p=0.05)$ however Dunn's post-test comparison between each group failed to show a significant difference. In addition iPOST did not alter the frequency of reperfusion arrhythmias $(p=0.16)$. When each characteristic was analysed using a two-way ANOVA, no interaction was seen between uraemia and iPOST except in the total duration of reperfusion arrhythmias when uraemia appeared to be associated with a reduction in arrhythmia duration $(\mathrm{p}=0.04)$, which was independent of iPOST.

\section{DISCUSSION}

Our data confirms previous reports that IPC is associated with a resistance to subsequent ischaemic and reperfusion arrhythmias in the absence of uraemia. ${ }^{9-12}$ In addition we have shown for the first time that uraemia does not lead to a rise in frequency or duration of arrhythmia occurring during ischaemic conditioning strategies.

This study failed to demonstrate a beneficial effect on arrhythmogenesis from RIPC or iPOST in either uraemic or non-uraemic animals in spite of our previous work, which demonstrated a marked reduction in infarct size using both these strategies. ${ }^{25}$

In this study we defined an arrhythmia as an alteration in the beat-to-beat blood pressure waveform so we were unable to distinguish atrial from ventricular arrhythmias. This limitation meant that we were unable to apply the Lambeth convention for the study of arrhythmias. ${ }^{26}$ In addition it may be argued that the use of beat-to-beat haemodynamic parameters as a surrogate for ventricular arrhythmias may lead to a misdiagnosis of ventricular arrhythmias, however we confirmed, in a small subset of animals, using contemporaneous ECG recording that the arrhythmias classified using haemodynamic parameters were caused by a ventricular arrhythmia. While further electrocardiographic studies would be helpful in differentiating atrial from ventricular arrhythmias in the uraemic heart, it is the haemodynamically significant arrhythmias (classically ventricular in origin), which are responsible for the high rates of sudden cardiac death and it is these arrhythmias that we are able to capture using our technique.

Documentation of arrhythmias associated specifically during IPC protocols have not been well described in the published literature, with many reports concentrating on arrhythmias

Table 2 The effect of three cycles of IPC on subsequent arrhythmia frequency and duration in a rodent model of chronic uraemia. SNx group $(\mathrm{n}=10)$ was compared with a SNx IPCX3 group $(\mathrm{n}=10)$.

\begin{tabular}{lccc}
\hline & SNx & SNx+IPCx3 & Value \\
\hline Duration of preconditioning ischaemic arrhythmias & $0(0-0)$ & $4(0-12)$ & 0.003 \\
Duration of preconditioning reperfusion arrhythmias & $0(0-0)$ & $46(20-50)$ & $2(0-5)$ \\
Duration of i25 ischaemic arrhythmias & $29(15-98)$ & $3(0-5)$ & 0.0001 \\
Duration of post i25 reperfusion arrhythmias & $9(7-19)$ & 0.003 \\
Total duration of ischaemic arrhythmias & $29(3-17)$ & 0.001 \\
Total duration of reperfusion arrhythmias & $9(7-19)$ & $48(25-55)$ & 0.03 \\
Total duration of all arrhythmias including preconditioning & $40(26-112)$ & $53(35-68)$ & $5(3-6)$ \\
Number of arrhythmias occurring during preconditioning & $0(0-0)$ & $0(0-1)$ & 0.002 \\
Number of i25 ischaemic arrhythmias & $2(3-3)$ & $0(0.0)$ & 0.87 \\
Number of post i25 reperfusion arrhythmias & $1(1-1)$ & $6(3-8)$ & 0.0001 \\
Total Number of all arrhythmias & $4(2-4)$ & 0.005 \\
\hline
\end{tabular}

i25 refers to the 25-min period of lethal ischaemia. Arrhythmias were defined as a haemodynamic disturbance in the mean arterial pressure (MAP) trace waveform lasting longer than

$2 \mathrm{~s}$ and occurring during each phase of the experimental protocol, with data displayed as median (IQR). Data (time in seconds) displayed as median (IQR) and $\mathrm{p}$ value calculated using Mann-Whitney U test.

IPC, ischaemic preconditioning; SNx, subtotal nephrectomy. 
Table 3 Table displaying the frequency and duration of arrhythmias (in seconds) between uraemic (SNx, n=10) and non-uraemic (Sham SNx, $\mathrm{n}=10$ ) animals undergoing one cycle of IPC followed by myocardial ischaemia-reperfusion

\begin{tabular}{|c|c|c|c|}
\hline & Sham SNx+IPC & SNx+IPC & p Value \\
\hline Duration of preconditioning ischaemic arrhythmias & $15(4-30)$ & $6(4-15)$ & 0.23 \\
\hline Duration of preconditioning reperfusion arrhythmias & $60(44-79)$ & $36(25-45)$ & 0.02 \\
\hline Duration of i35 ischaemic arrhythmias & $8(2-18)$ & $6(0-16)$ & 0.74 \\
\hline Duration post i35 reperfusion arrhythmias & $0(0-10)$ & $0(0-6)$ & 0.64 \\
\hline Total duration of ischaemic arrhythmias & $30(10-43)$ & $16(8-24)$ & 0.22 \\
\hline Total duration of reperfusion arrhythmias & $62(44-88)$ & $38(29-47)$ & 0.01 \\
\hline Total duration of all arrhythmias including preconditioning & $81(68-144)$ & $56(41-69)$ & 0.02 \\
\hline Number of arrhythmias occurring during preconditioning & $3(2-4)$ & $2(1-3)$ & 0.37 \\
\hline Number of i25 ischaemic arrhythmias & $2(1-3)$ & $1(0-1)$ & 0.32 \\
\hline Number of post i25 reperfusion arrhythmias & $1(0-1)$ & $0(0-1)$ & 0.18 \\
\hline Total number of all arrhythmias & $6(3-7)$ & $4(3-6)$ & 0.27 \\
\hline
\end{tabular}

i25 refers to the 25 -min period of lethal ischaemia and i35 refers to the 35-min period of lethal ischaemia. Arrhythmias were defined as a haemodynamic disturbance in the mean arterial pressure (MAP) trace waveform lasting longer than $2 \mathrm{~s}$ and occurring during each phase of the experimental protocol, with data displayed as median (IQR). Data displayed as median (IQR), with $\mathrm{p}$ value calculated using Mann-Whitney test.

IPC, ischaemic preconditioning; SNx, subtotal nephrectomy.

occurring during the standard ischaemia-reperfusion phase which occurred after the IPC protocol. When the arrhythmias associated with the preconditioning cycles were added to those occurring during the ischaemia-reperfusion phase the seemingly antiarrhythmic effect of IPC was lost, in terms of the absolute number of arrhythmias and their total duration. In light of this observation a novel way to describe the effects of IPC on myocardial arrhythmias would be that IPC shifts the arrhythmias forward in time from the lethal ischaemic and reperfusion phase to the IPC phase. Whether or not this change in the timing of arrhythmias has any direct effect on subsequent tissue protection is unclear. However hypotension resulting from ventricular arrhythmias occurring during IPC may be a part of the tissue protective signal. Indeed there have been several studies, which have shown tissue protection similar to IPC following episodes of temporary pacing ${ }^{27}$ or induction of ventricular fibrillation $^{29}$ through demand ischaemia and loss of cardiac output, respectively. The additional benefit of these arrhythmias above and beyond the direct stimulation of the cytoprotective pathway by ischaemia in subsequent tissue protection is unknown. However this may explain the finding that IPC is a more potent cytoprotective strategy than either RIPC or iPOST which is borne out in our previous work which demonstrated that although there was no difference in the area at risk there was a relative reduction in infarct size of $86 \%$ in IPC, 59\% in RIPC and $47 \%$ in iPOST. $^{25}$ Arrhythmia generation from IPC is however unlikely to be the main trigger for subsequent tissue protection because IPC-induced arrhythmia suppression can be pharmacologically divorced from tissue protection ${ }^{10} 30$ and IPC is known to confer resistance to ischaemia in all the organs studied rather than just the myocardium.

The mechanism of arrhythmia suppression following ischaemic conditioning is not fully understood: it does not appear to be mediated by adenosine, bradykinin or prostaglandin ${ }^{30}$ or via depletion of endogenous catecholamines ${ }^{31}$ however there is evidence of involvement of the mitochondrial $\mathrm{K}_{\mathrm{ATP}}$ channel and reactive oxygen species in the antiarrhythmic effects seen with IPC. ${ }^{32}$ Further work is required to investigate in detail the antiarrhythmic effects of IPC, RIPC and iPOST.

These results demonstrate that contrary to expectations uraemia did not lead to increased arrhythmia frequency or duration during myocardial ischaemia/reperfusion. Indeed our results suggest that underlying uraemia may lead to a reduction in reperfusion arrhythmia duration seen in the context of one cycle of IPC and during iPOST, but not during RIPC. However no statistically significant differences in arrhythmia frequency or duration were seen during the ischaemic phase. This finding is in contrast to epidemiological data, which report that uraemia is a risk factor for myocardial dysrhythmias leading to a high incidence of sudden cardiac death following a myocardial event. ${ }^{33}$

A potential explanation for this discrepancy could be that the four-week SNx model does not demonstrate the cardiac phenotype seen in patients with CKD. However this four-week model has been shown to develop hypertension, ${ }^{25}{ }^{34} \mathrm{LVH}^{34}{ }^{35}$ a reduced fractional shortening on echocardiography, ${ }^{36}$ impaired cardiac function secondary to bioenergetic failure, ${ }^{35}$ capillary

Table 4 The effect of RIPC in uraemic and non-uraemic animals on the frequency and duration of arrhythmias

\begin{tabular}{|c|c|c|c|c|c|}
\hline & Sham SNx & Sham SNx + RIPC & SNx & SNx + RIPC & p Value \\
\hline Duration of ischaemic arrhythmias & 65 (24-134) & $35(20-85)$ & $30(11-115)$ & $45(16-71)$ & 0.74 \\
\hline Duration of reperfusion arrhythmias & $15(8-22)$ & $14(4-17)$ & $9(5-19)$ & $11(4-11)$ & 0.5 \\
\hline Total duration of all arrhythmias & $73(37-151)$ & $52(35-101)$ & $30(24-142)$ & $52(40-74)$ & 0.54 \\
\hline Number of arrhythmias occurring during ischaemia & $1(0-4)$ & $1(0-3)$ & $1(0-3)$ & $1(0-3)$ & 0.89 \\
\hline Number of arrhythmias occurring during reperfusion & $0(0-1)$ & $0(0-1)$ & $1(0-1)$ & $0(0-1)$ & 0.64 \\
\hline Total Number of all arrhythmias & $2(1-4)$ & $1(0-3)$ & $2(1-4)$ & $2(0-2)$ & 0.79 \\
\hline
\end{tabular}

Four groups were studied; a non-uraemic group (Sham $S N x, n=10)$, an uraemic group $(S N x, n=10)$, a non-uraemic group which underwent a RIPC protocol (Sham $S N x+R I P C, n=9)$ and an uraemic group that underwent a RIPC protocol $(S N x+R I P C, n=8)$. Data displayed as median (IQR), with $p$ value calculated using a two-tailed Mann-Whitney $U$ test.

$\mathrm{RIPC}$, remote ischaemic preconditioning; SNx, subtotal nephrectomy. 
Table 5 The effect of iPOST in uraemic and non-uraemic animals on arrhythmia frequency and duration

\begin{tabular}{|c|c|c|c|c|c|}
\hline & Sham SNx & Sham SNx iPOST & SNx & SNx iPOST & p Value \\
\hline Duration of ischaemic arrhythmias & $88(42-232)$ & $163(37-314)$ & $70(3-124)$ & $73(50-175)$ & 0.36 \\
\hline Duration of reperfusion arrhythmias & $16(7-21)$ & $21(13-63)$ & $11(8-19)$ & $15(2-20)$ & 0.05 \\
\hline Total duration of all arrhythmias & $104(46-244)$ & $202(6-345)$ & $78(30-146)$ & $85(58-181)$ & 0.23 \\
\hline Number of arrhythmias occurring during ischaemia & $3(1-4)$ & $2(1-4)$ & $3(0-3)$ & $1(1-3)$ & 0.27 \\
\hline Number of arrhythmias occurring during reperfusion & $1(0-1)$ & $1(1-2)$ & $1(0-1)$ & $1(0-1)$ & 0.16 \\
\hline Total Number of all arrhythmias & $3(2-5)$ & $4(2-5)$ & $3(1-5)$ & $2(1-4)$ & 0.79 \\
\hline
\end{tabular}

Four groups were studied; a non-uraemic group (Sham $S N x, n=9$ ), an uraemic group (SNx, n=9), a non-uraemic group which underwent an iPOST protocol (Sham SNx iPOST, $n=8$ ) and an uraemic group, which underwent an iPOST protocol (SNx iPOST, $n=9$ ). Data displayed as median (IQR), with $p$ value calculated using a two-tailed Mann-Whitney U test. iPOST, ischaemic postconditioning; SNx, subtotal nephrectomy.

rarefaction and myocardial interstitial cell proliferation ${ }^{37}$ leading to reduced ischaemia tolerance. ${ }^{25}{ }^{34}$ Additionally even if the SNx model did not represent a severe uraemic phenotype, it is known that even mild to moderate CKD leads to a huge burden of cardiovascular disease: results from the HOPE study indicate that patients with moderate CKD (serum creatinine $125-200 \mu \mathrm{mol} / \mathrm{L}$ ) had a $40 \%$ increase in sudden cardiac death when compared with individuals with normal renal function, ${ }^{38}$ that patients with CKD stage 3 had an OR for cardiovascular events, of two to four compared patients with normal renal function and up to $40 \%$ of patients presenting with a myocardial infarction have mild to moderate renal failure. ${ }^{39}$

The increased incidence of sudden cardiac death in the CKD cohort could be explained by the increased incidence of acute myocardial events triggering arrhythmias and that the arrhythmias associated with sudden cardiac death are by definition ischaemic, rather than reperfusion driven and our data does not show any difference in ischaemia driven arrhythmia frequency or duration in the context of uraemia. An explanation for both the epidemiological data, which suggests that uraemia predisposes to malignant ventricular arrhythmias and our animal data, which found no increase in the susceptibility to ischaemic arrhythmias would be to conclude that that the increased incidence of cardiac dysrhythmias seen in people with CKD may be driven primarily by an increased incidence of ischaemic events rather than by an increase in the susceptibility to arrhythmias.

In conclusion these data demonstrate that, compared to a non-uraemic heart, the uraemic heart does not have a lower threshold severe arrhythmias and has a similar antiarrhythmic response to ischaemic conditioning strategies.

Contributors $\mathrm{KM}, \mathrm{CJB}, \mathrm{JK}$ and MMY: Conception or design, or analysis and interpretation of data, or both. KM, CJB, JK, MR, CT and MMY: Drafting the article or revising it. $\mathrm{KM}, \mathrm{CJB}, \mathrm{MR}, \mathrm{CT}$ and MMY: Providing intellectual content of critical importance to the work described. KM, CJB, JK, MR, CT and MMY: Final approval of the version to be published.

Funding Departmental.

Competing interests None.

Provenance and peer review Not commissioned; externally peer reviewed.

\section{REFERENCES}

1 Collins AJ, Foley RN, Chavers B, et al. 'United States Renal Data System 2011 Annual Data Report: Atlas of chronic kidney disease \& end-stage renal disease in the United States. Am J Kidney Dis 2012;59(1 Suppl 1):A7, e1-420.

2 Pun PH, Smarz TR, Honeycutt EF, et al. Chronic kidney disease is associated with increased risk of sudden cardiac death among patients with coronary artery disease. Kidney Int 2009;76:652-8.

3 Parekh RS, Plantinga LC, Kao WH, et al. The association of sudden cardiac death with inflammation and other traditional risk factors. Kidney Int 2008;74:1335-42.

4 Pun PH, Lehrich RW, Honeycutt EF, et al. Modifiable risk factors associated with sudden cardiac arrest within hemodialysis clinics. Kidney Int 2011;79:218-27.
5 Shamseddin MK, Parfrey PS. Sudden cardiac death in chronic kidney disease: epidemiology and prevention. Nat Rev Nephrol 2011;7:145-54.

6 Murry $C E$, Jennings RB, Reimer KA. Preconditioning with ischemia: a delay of lethal cell injury in ischemic myocardium. Circulation 1986;74:1124-36.

7 Przyklenk K, Bauer B, Ovize M, et al. Regional ischemic 'preconditioning' protects remote virgin myocardium from subsequent sustained coronary occlusion. Circulation 1993:87:893-9.

8 Zhao ZQ, Corvera JS, Halkos M, et al. Inhibition of myocardial injury by ischemic postconditioning during reperfusion: comparison with ischemic preconditioning. Am J Physiol Heart Circ Physiol 2003;285:H579-88.

9 Hagar JM, Hale SL, Kloner RA. Effect of preconditioning ischemia on reperfusion arrhythmias after coronary artery occlusion and reperfusion in the rat. Circ Res 1991:68:61-8.

10 Ravingerova T, Matejikova J, Neckar J, et al. Differential role of PI3K/Akt pathway in the infarct size limitation and antiarrhythmic protection in the rat heart. Mol Cell Biochem 2007;297:111-20.

11 Driamov SV, Bellahcene M, Butz S, et al. Bradykinin is a mediator, but unlikely a trigger, of antiarrhythmic effects of ischemic preconditioning. J Cardiovasc Electrophysiol 2007;18:93-9.

12 Das B, Sarkar C. Similarities between ischemic preconditioning and 17beta-estradiol mediated cardiomyocyte KATP channel activation leading to cardioprotective and antiarrhythmic effects during ischemia/reperfusion in the intact rabbit heart. J Cardiovasc Pharmacol 2006;47:277-86.

13 Buyukates $\mathrm{M}$, Kalaycioglu $\mathrm{S}, \mathrm{Oz} \mathrm{E}$, et al. Effects of ischemic preconditioning in human heart. J Card Surg 2005;20:241-5.

14 Wu ZK, livainen T, Pehkonen E, et al. Perioperative and postoperative arrhythmia in three-vessel coronary artery disease patients and antiarrhythmic effects of ischemic preconditioning. Eur J Cardiothorac Surg 2003;23:578-84.

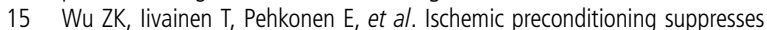
ventricular tachyarrhythmias after myocardial revascularization. Circulation 2002;106:3091-6.

16 Grund F, Sommerschild HT, Kirkeboen KA, et al. Proarrhythmic effects of ischemic preconditioning in anesthetized pigs. Basic Res Cardiol 1997;92:417-25.

17 Jebeli M, Esmaili HR, Mandegar MH, et al. Evaluation of the effects of ischemic preconditioning with a short reperfusion phase on patients undergoing a coronary artery bypass graft. Ann Thorac Cardiovasc Surg 2010;16:248-52.

18 Hajrasouliha AR, Tavakoli S, Ghasemi M, et al. Endogenous cannabinoids contribute to remote ischemic preconditioning via cannabinoid $C B 2$ receptors in the rat heart. Eur J Pharmacol 2008;579:246-52.

19 Schmidt MR, Smerup M, Konstantinov IE, et al. Intermittent peripheral tissue ischemia during coronary ischemia reduces myocardial infarction through a KATP-dependent mechanism: first demonstration of remote ischemic perconditioning. Am J Physiol Heart Circ Physiol 2007;292:H1883-90.

20 Li SJ, Wu YN, Kang Y, et al. Noninvasive Limb Ischemic Preconditioning Protects Against Myocardial I/R Injury in Rats. J Surg Res 2009;164:162-8.

21 Lujan HL, DiCarlo SE. Partial hindlimb occlusion reduced the susceptibility to sustained ventricular tachycardia in conscious rats. J Cardiovasc Pharmacol Ther 2009;14:199-206.

22 Galagudza M, Kurapeev D, Minasian S, et al. Ischemic postconditioning: brief ischemia during reperfusion converts persistent ventricular fibrillation into regular rhythm. Eur J Cardiothorac Surg 2004;25:1006-10.

23 Dow J, Bhandari A, Kloner RA. The mechanism by which ischemic postconditioning reduces reperfusion arrhythmias in rats remains elusive. J Cardiovasc Pharmacol Ther 2009;14:99-103.

24 Lauzier B, Sicard P, Bouchot O, et al. After four hours of cold ischemia and cardioplegic protocol, the heart can still be rescued with postconditioning. Transplantation 2007;84:1474-82.

25 Byrne CJ, McCafferty K, Kieswich J, et al. Ischemic conditioning protects the uremic heart in a rodent model of myocardial infarction. Circulation 2012;125:1256-65. 
26 Curtis MJ, Hancox JC, Farkas A, et al. The Lambeth Conventions (II): guidelines for the study of animal and human ventricular and supraventricular arrhythmias. Pharmacol Ther 2013;139:213-48.

27 Vegh A, Szekeres L, Parratt JR. Transient ischaemia induced by rapid cardiac pacing results in myocardial preconditioning. Cardiovasc Res 1991:25:1051-3.

28 Vanagt WY, Cornelussen RN, Poulina QP, et al. Pacing-induced dys-synchrony preconditions rabbit myocardium against ischemia/reperfusion injury. Circulation 2006;114(1 Suppl):I264-9.

29 Hearse DJ, Ferrari R, Sutherland FJ. Cardioprotection: intermittent ventricular fibrillation and rapid pacing can induce preconditioning in the blood-perfused rat heart. J Mol Cell Cardiol 1999;31:1961-73.

30 Miura T, Ishimoto R, Sakamoto J, et al. Suppression of reperfusion arrhythmia by ischemic preconditioning in the rat: is it mediated by the adenosine receptor prostaglandin, or bradykinin receptor? Basic Res Cardiol 1995; 90:240-6.

31 Lawson CS, Hearse DJ. Anti-arrhythmic protection by ischaemic preconditioning in isolated rat hearts is not due to depletion of endogenous catecholamines. Cardiovasc Res 1996;31:655-62.

32 Matejikova J, Kucharska J, Pinterova $M$, et al. Protection against ischemia-induced ventricular arrhythmias and myocardial dysfunction conferred by preconditioning in the rat heart: involvement of mitochondrial K(ATP) channels and reactive oxygen species. Physiol Res 2009;58:9-19.

33 Herzog CA, Strief JW, Collins AJ, et al. Cause-specific mortality of dialysis patients after coronary revascularization: why don't dialysis patients have better survival after coronary intervention? Nephrol Dial Transplant 2008;23:2629-33.

34 Dikow $\mathrm{R}$, Kihm LP, Zeier $\mathrm{M}$, et al. Increased infarct size in uremic rats: reduced ischemia tolerance? J Am Soc Nephrol 2004;15:1530-6.

35 Raine $A E$, Seymour $A M$, Roberts $A F$, et al. Impairment of cardiac function and energetics in experimental renal failure. J Clin Invest 1993;92:2934-40.

36 Koleganova N, Piecha G, Ritz E, et al. Interstitial fibrosis and microvascular disease of the heart in uremia: amelioration by a calcimimetic. Lab Invest 2009;89:520-30.

37 Amann K, Odoni G, Benz K, et al. Sympathetic blockade prevents the decrease in cardiac VEGF expression and capillary supply in experimental renal failure. Am J Physiol Renal Physiol 2011;300:F105-12.

38 Mann JF, Gerstein HC, Pogue J, et al. Renal insufficiency as a predictor of cardiovascular outcomes and the impact of ramipril: the HOPE randomized trial. Ann Intern Med 2001;134:629-36.

39 Wright RS, Reeder GS, Herzog CA, et al. Acute myocardial infarction and renal dysfunction: a high-risk combination. Ann Intern Med 2002;137:563-70. 